\title{
A New Analytical Study of Modified Camassa-Holm and Degasperis-Procesi Equations
}

\author{
Majeed A. Yousif1', Bewar A. Mahmood ${ }^{2}$, Fadhil H. Easif ${ }^{1}$ \\ ${ }^{1}$ Department of Mathematics, University of Zakho, Zakho, Iraq \\ ${ }^{2}$ Department of Mathematics, University of Duhok, Duhok, Iraq \\ Email: majeed.ahmed@uod.ac, bewar.ahmed@uod.ac, dean.sic@uoz.ac
}

Received 23 June 2015; accepted 28 August 2015; published 31 August 2015

Copyright (C) 2015 by authors and Scientific Research Publishing Inc.

This work is licensed under the Creative Commons Attribution International License (CC BY). http://creativecommons.org/licenses/by/4.0/

c) (7) Open Access

\begin{abstract}
In this letter, variational homotopy perturbation method (VHPM) has been studied to obtain solitary wave solutions of modified Camassa-Holm and Degasperis-Procesi equations. The results show that the VHPM is suitable for solving nonlinear differential equations with fully nonlinear dispersion term. The travelling wave solution for above equation compared with VIM, HPM, and exact solution. Also, it was shown that the present method is effective, suitable, and reliable for these types of equations.
\end{abstract}

\section{Keywords}

Homotopy Perturbation Method, Modified Camassa-Holm Equation, Modified Degasperis-Procesi Equation

\section{Introduction}

Many varieties of physical, chemical, and biological phenomena can be expressed in terms of nonlinear partial differential equations. In most cases, it is difficult to obtain the exact solution for these equations. Therefore analytical methods have been used to find approximate solutions. In recent years, many analytical methods such as the Adomian decomposition method [1] [2], the homotopy analysis method [3] [4], the variational iteration method [5] [6], the homotopy perturbation method [7]-[10], and variational homotopy perturbation method [11] [12] have been utilized to solve linear and nonlinear equations.

In this paper, we will use variational homotopy perturbation method to study the Modified Camassa-Holm and Degasperis-Procesi equations and obtain their analytical solutions. 


\section{Mathematical Models}

Wazwaz [13] studies a family of important physically equations which is called modified $b$-equation. It has the following expression:

$$
u_{t}-u_{x x t}+(b+1) u^{2} u_{x}-b u_{x} u_{x x}-u u_{x x x}=0 .
$$

where $b$ is a positive integer. As is known, when $b=2, b=3$, Equation (1) reduces to modified CamassaHolm $(\mathrm{mCH})$ equation and modified Degasperis-Procesi (mDP) equation, respectively.

The $\mathrm{mCH}$ equation with exact solution [13]:

$$
\begin{gathered}
u_{t}-u_{x x t}+3 u^{2} u_{x}-2 u_{x} u_{x x}-u u_{x x x}=0, \\
u(x, t)=-2 \operatorname{sech}^{2}\left(\frac{1}{2} x-t\right) .
\end{gathered}
$$

The mDP equation with exact solution [13]:

$$
\begin{gathered}
u_{t}-u_{x x t}+4 u^{2} u_{x}-3 u_{x} u_{x x}-u u_{x x x}=0 \\
u(x, t)=-\frac{15}{8} \operatorname{sech}^{2}\left(\frac{1}{2} x-\frac{5}{4} t\right)
\end{gathered}
$$

\section{Analytical Methods}

\subsection{Variational Iteration Method (VIM)}

To clarify the basic ideas of VIM, we consider the following differential equation

$$
L u+N u=g(x, t) .
$$

where $L$ is a linear operator defined by $L=\frac{\partial^{m}}{\partial t^{m}}, m \in \mathbb{N}, N$ is a nonlinear operator and $g(x, t)$ is a known analytic function. According to (VIM), we can write down a correction functional as follows:

$$
u_{n+1}(x, t)=u_{n}(x, t)+\int_{0}^{t} \lambda\left(L u_{n}(x, \tau)+N \tilde{u}_{n}(x, \tau)-g(x, \tau)\right) \mathrm{d} \tau .
$$

where $\lambda$ is a general lagrangian multiplier by [14] defined as:

$$
\lambda(x, \tau)=\frac{(-1)^{m}}{(m-1) !}(\tau-t)^{m-1}, m \geq 1 .
$$

The subscript $n$ indicates the $n$th approximation and $\tilde{u}_{n}$ is considered as a restricted variation [15].

\subsection{Homotopy Perturbation Method (HPM)}

To illustrate the basic idea of this method, we consider the following nonlinear differential equation:

$$
A(u)-f(r)=0, \quad r \in \Omega .
$$

with the boundary condition

$$
B\left(u, \frac{\partial u}{\partial n}\right)=0 \quad r \in \Gamma .
$$

where $A$ is a general differential operator, $B$ a boundary operator, $f(r)$ a known analytical function and $\Gamma$ is the boundary of the domain $\Omega$. A can be divided into two parts which are $L$ and $N$, where $L$ is linear and $N$ is nonlinear. Equation (9) can therefore be rewritten as follows:

$$
L(u)+N(u)-f(r)=0, \quad r \in \Omega .
$$

Homotopy perturbation structure is shown as follows: 


$$
H(v, p)=(1-p)\left[L(v)-L\left(u_{0}\right)\right]+p[A(v)-f(r)]=0,
$$

where

$$
v(r, p): \Omega \times[0,1] \rightarrow R
$$

In Equation (12), $p \in[0,1]$ is an embedding parameter and $u_{0}$ is the first approximation that satisfies the boundary condition. We can assume that the solution of Equation (12) can be written as a power series in $p$, as following:

$$
v=v_{0}+p v_{1}+p^{2} v_{2}+\cdots
$$

and the best approximation for solution is:

$$
u=\lim _{p \rightarrow 0} v=v_{0}+v_{1}+v_{2}+\cdots .
$$

It is well known that series (15) is convergent for most of the cases and also the rate of convergence depends on $L(u)$. We assume that Equation (15) has a unique solution [7].

\subsection{Variational Homotopy Perturbation Method (VHPM)}

To illustrate the concept of the variational homotopy perturbation method, we consider the general differential Equation (6). We construct the correction functional (7) and apply the homotopy perturbation method (14) to obtain:

$$
\sum_{n=0}^{\infty} p^{n} u_{n}(x, t)=u_{0}(x, t)+p \int_{0}^{t} \lambda(x, \tau)\left(\sum_{n=0}^{\infty} p^{n} L u_{n}(x, \tau)+N \sum_{n=0}^{\infty} p^{n} \tilde{u}_{n}(x, \tau)-g(x, \tau)\right) \mathrm{d} \tau .
$$

As we see, the procedure is formulated by the coupling of variational iteration method and homotopy perturbation method. A comparison of like powers of $p$ gives solutions of various orders.

\section{Application of VHPM}

In this section, we apply the variational homotopy perturbation method to solve $\mathrm{mCH}$ and mDP equations.

\subsection{Application of VHPM to Modified Camassa-Holm Equation}

Consider the $\mathrm{mCH}$ equation

$$
\begin{gathered}
u_{t}-u_{x x t}+3 u^{2} u_{x}-2 u_{x} u_{x x}-u u_{x x x}=0 \\
u(x, 0)=-2 \operatorname{sech}^{2}\left(\frac{1}{2} x\right)
\end{gathered}
$$

To solve Equation (17), using VIM, we have the correction functional as:

$$
u_{n+1}(x, t)=u_{n}(x, t)+\int_{0}^{t} \lambda\left(\left(u_{n}\right)_{\tau}-\left(\tilde{u}_{n}\right)_{x x \tau}+3\left(\tilde{u}_{n}\right)^{2}\left(\tilde{u}_{n}\right)_{x}-2\left(\tilde{u}_{n}\right)_{x}\left(\tilde{u}_{n}\right)_{x x}-\tilde{u}_{n}\left(\tilde{u}_{n}\right)_{x x x}\right) \mathrm{d} \tau,
$$

where $\tilde{u}_{n}$ is considered as a restricted variation. Making the above functional stationary, the Lagrange multiplier can be determined as Equation (8) is $\lambda=-1$, which yields the following iteration formula:

$$
u_{n+1}(x, t)=u_{n}(x, t)-\int_{0}^{t}\left(\left(u_{n}\right)_{\tau}-\left(u_{n}\right)_{x x \tau}+3\left(u_{n}\right)^{2}\left(u_{n}\right)_{x}-2\left(u_{n}\right)_{x}\left(u_{n}\right)_{x x}-u_{n}\left(u_{n}\right)_{x x x}\right) \mathrm{d} \tau .
$$

Applying the variational homotopy perturbation method, we have:

$$
\begin{aligned}
\sum_{n=0}^{\infty} p^{n} u_{n}(x, t)= & u_{0}(x, t)-p \int_{0}^{t}\left[\left(\sum_{n=0}^{\infty} p^{n} u_{n}\right)_{\tau}-\left(\sum_{n=0}^{\infty} p^{n} u_{n}\right)_{x x \tau}+3\left(\sum_{n=0}^{\infty} p^{n} u_{n}\right)^{2}\left(\sum_{n=0}^{\infty} p^{n} u_{n}\right)_{x}\right. \\
& \left.-2\left(\sum_{n=0}^{\infty} p^{n} u_{n}\right)_{x}\left(\sum_{n=0}^{\infty} p^{n} u_{n}\right)_{x x}-\left(\sum_{n=0}^{\infty} p^{n} u_{n}\right)\left(\sum_{n=0}^{\infty} p^{n} u_{n}\right)_{x x x}\right] \mathrm{d} \tau,
\end{aligned}
$$


Substituting initial condition (18)

$$
\begin{aligned}
\sum_{n=0}^{\infty} p^{n} u_{n}(x, t)= & -2 \operatorname{sech}^{2}\left(\frac{1}{2} x\right)-p \int_{0}^{t}\left[\left(\sum_{n=0}^{\infty} p^{n} u_{n}\right)_{\tau}-\left(\sum_{n=0}^{\infty} p^{n} u_{n}\right)_{x x \tau}+3\left(\sum_{n=0}^{\infty} p^{n} u_{n}\right)^{2}\left(\sum_{n=0}^{\infty} p^{n} u_{n}\right)_{x}\right. \\
& \left.-2\left(\sum_{n=0}^{\infty} p^{n} u_{n}\right)_{x}\left(\sum_{n=0}^{\infty} p^{n} u_{n}\right)_{x x}-\left(\sum_{n=0}^{\infty} p^{n} u_{n}\right)\left(\sum_{n=0}^{\infty} p^{n} u_{n}\right)_{x x x}\right] \mathrm{d} \tau .
\end{aligned}
$$

Comparing the coefficient of like powers of $p$, we have

$$
\begin{gathered}
p^{0}: u_{0}(x, t)=-2 \operatorname{sech}^{2}\left(\frac{1}{2} x\right), \\
p^{1}: u_{1}(x, t)=-\int_{0}^{t}\left[\left(u_{0}\right)_{\tau}-\left(u_{0}\right)_{x x \tau}+3\left(u_{0}\right)^{2}\left(u_{0}\right)_{x}-2\left(u_{0}\right)_{x}\left(u_{0}\right)_{x x}-u_{0}\left(u_{0}\right)_{x x x}\right] \mathrm{d} \tau, \\
p^{2}: u_{2}(x, t)=-\int_{0}^{t}\left[\left(u_{1}\right)_{\tau}-\left(u_{1}\right)_{x x \tau}+3\left(\left(u_{0}\right)^{2}\left(u_{1}\right)_{x}+2\left(u_{0}\right)_{x}\left(u_{0}\right)\left(u_{1}\right)\right)\right. \\
\left.-2\left(\left(u_{0}\right)_{x}\left(u_{1}\right)_{x x}+\left(u_{1}\right)_{x}\left(u_{0}\right)_{x x}\right)\left(u_{0}\left(u_{1}\right)_{x x x}+u_{1}\left(u_{0}\right)_{x x x}\right)\right] \mathrm{d} \tau,
\end{gathered}
$$

Then

$$
\begin{gathered}
u_{0}(x, t)=-2 \operatorname{sech}^{2}\left(\frac{1}{2} x\right), \\
u_{1}(x, t)=-12 t \operatorname{sech}^{4}\left(\frac{1}{2} x\right) \tanh \left(\frac{1}{2} x\right) .
\end{gathered}
$$

For an arbitrary $u_{j}(x, t)(j=2,3, \cdots)$ we can use symbolic software programme such as Mathematica to calculate it in the same manner.

If only the two-term approximation of Equation (15) is sufficient, then the approximate solution of Equation (17) will be expressed as:

$$
u(x, t)=-2 \operatorname{sech}^{2}\left(\frac{1}{2} x\right)-12 \operatorname{sech}^{4}\left(\frac{1}{2} x\right) \tanh \left(\frac{1}{2} x\right) .
$$

From its expression one can see that it is also a solitary wave solution.

Remark 1. It should be remarked that the graph drawn here and approximate solution using VHPM is in excellent agreement with HPM [16] and VIM [17].

\subsection{Application of VHPM to Modified Degasperis-Procesi}

Consider the mDP equation

$$
\begin{gathered}
u_{t}-u_{x x t}+4 u^{2} u_{x}-3 u_{x} u_{x x}-u u_{x x x}=0, \\
u(x, 0)=-\frac{15}{8} \operatorname{sech}^{2}\left(\frac{x}{2}\right) .
\end{gathered}
$$

To solve Equation (29), using VIM, we have the correction functional as:

$$
u_{n+1}(x, t)=u_{n}(x, t)+\int_{0}^{t} \lambda\left(\left(u_{n}\right)_{\tau}-\left(\tilde{u}_{n}\right)_{x x \tau}+4\left(\tilde{u}_{n}\right)^{2}\left(\tilde{u}_{n}\right)_{x}-3\left(\tilde{u}_{n}\right)_{x}\left(\tilde{u}_{n}\right)_{x x}-\tilde{u}_{n}\left(\tilde{u}_{n}\right)_{x x x}\right) \mathrm{d} \tau,
$$

where $\tilde{u}_{n}$ is considered as a restricted variation. Making the above functional stationary, the Lagrange multiplier can be determined as Equation (8) is $\lambda=-1$, which yields the following iteration formula:

$$
u_{n+1}(x, t)=u_{n}(x, t)-\int_{0}^{t}\left(\left(u_{n}\right)_{\tau}-\left(u_{n}\right)_{x x \tau}+4\left(u_{n}\right)^{2}\left(u_{n}\right)_{x}-3\left(u_{n}\right)_{x}\left(u_{n}\right)_{x x}-u_{n}\left(u_{n}\right)_{x x x}\right) \mathrm{d} \tau .
$$

Applying the variational homotopy perturbation method, we have: 


$$
\begin{aligned}
\sum_{n=0}^{\infty} p^{n} u_{n}(x, t)= & u_{0}(x, t)-p \int_{0}^{t}\left[\left(\sum_{n=0}^{\infty} p^{n} u_{n}\right)_{\tau}-\left(\sum_{n=0}^{\infty} p^{n} u_{n}\right)_{x x \tau}\right. \\
& +4\left(\sum_{n=0}^{\infty} p^{n} u_{n}\right)^{2}\left(\sum_{n=0}^{\infty} p^{n} u_{n}\right)_{x}-3\left(\sum_{n=0}^{\infty} p^{n} u_{n}\right)_{x}\left(\sum_{n=0}^{\infty} p^{n} u_{n}\right)_{x x} \\
& \left.-\left(\sum_{n=0}^{\infty} p^{n} u_{n}\right)\left(\sum_{n=0}^{\infty} p^{n} u_{n}\right)_{x x x}\right] \mathrm{d} \tau,
\end{aligned}
$$

Substituting initial condition (30)

$$
\begin{aligned}
\sum_{n=0}^{\infty} p^{n} u_{n}(x, t)= & -\frac{15}{8} \operatorname{sech}^{2}\left(\frac{x}{2}\right)-p \int_{0}^{t}\left[\left(\sum_{n=0}^{\infty} p^{n} u_{n}\right)_{\tau}-\left(\sum_{n=0}^{\infty} p^{n} u_{n}\right)_{x x \tau}+3\left(\sum_{n=0}^{\infty} p^{n} u_{n}\right)^{2}\left(\sum_{n=0}^{\infty} p^{n} u_{n}\right)_{x}\right. \\
& \left.-2\left(\sum_{n=0}^{\infty} p^{n} u_{n}\right)_{x}\left(\sum_{n=0}^{\infty} p^{n} u_{n}\right)_{x x}-\left(\sum_{n=0}^{\infty} p^{n} u_{n}\right)\left(\sum_{n=0}^{\infty} p^{n} u_{n}\right)_{x x x}\right] \mathrm{d} \tau .
\end{aligned}
$$

Comparing the coefficient of like powers of $p$, we have

$$
\begin{gathered}
p^{0}: u_{0}(x, t)=-\frac{15}{8} \operatorname{sech}^{2}\left(\frac{x}{2}\right) \\
p^{1}: u_{1}(x, t)=-\int_{0}^{t}\left[\left(u_{0}\right)_{\tau}-\left(u_{0}\right)_{x x \tau}+4\left(u_{0}\right)^{2}\left(u_{0}\right)_{x}-3\left(u_{0}\right)_{x}\left(u_{0}\right)_{x x}-u_{0}\left(u_{0}\right)_{x x x}\right] \mathrm{d} \tau, \\
p^{2}: u_{2}(x, t)=-\int_{0}^{t}\left[\left(u_{1}\right)_{\tau}-\left(u_{1}\right)_{x x \tau}+4\left(\left(u_{0}\right)^{2}\left(u_{1}\right)_{x}+2\left(u_{0}\right)_{x}\left(u_{0}\right)\left(u_{1}\right)\right)\right. \\
\left.-3\left(\left(u_{0}\right)_{x}\left(u_{1}\right)_{x x}+\left(u_{1}\right)_{x}\left(u_{0}\right)_{x x}\right)-\left(u_{0}\left(u_{1}\right)_{x x x}+u_{1}\left(u_{0}\right)_{x x x}\right)\right] \mathrm{d} \tau,
\end{gathered}
$$

Then

$$
\begin{gathered}
u_{0}(x, t)=-\frac{15}{8} \operatorname{sech}^{2}\left(\frac{x}{2}\right), \\
u_{1}(x, t)=-\frac{225}{16} t \operatorname{sech}^{4}\left(\frac{1}{2} x\right) \tanh \left(\frac{1}{2} x\right) .
\end{gathered}
$$

For an arbitrary $u_{j}(x, t)(j=2,3, \cdots)$ we can use symbolic software programme such as Mathematica to calculate it in the same manner. If only the two-term approximation of Equation (15) is sufficient, then the approximate solution of Equation (29) will be expressed as:

$$
u(x, t)=-\frac{15}{8} \operatorname{sech}^{2}\left(\frac{x}{2}\right)-\frac{225}{16} t \operatorname{sech}^{4}\left(\frac{1}{2} x\right) \tanh \left(\frac{1}{2} x\right) .
$$

From its expression one can see that it is also a solitary wave solution.

Remark 2. It should be remarked that the graph drawn here and approximate solution using VHPM is in excellent agreement with HPM [16] and VIM [17].

\section{Figures}

In this section, we show the accurance of VHPM to finding analytical solution of Modified Camassa-Holm and Degasperis-Procesi equations. Also, we compare between exact and analytical solution (see Figures 1-3).

\section{Conclusion}

In this paper, we apply variational homotopy perturbation method to obtain the analytical solutions of Modified Camassa-Holm and Degasperis-Procesi equations. The solutions obtained by present method is compared with the exact solution. Also, it was shown that the approximation solution by VHPM had a good agreement with HPM and VIM. We observed that the method is effective for given examples and it can be applied to many other nonlinear equations. 


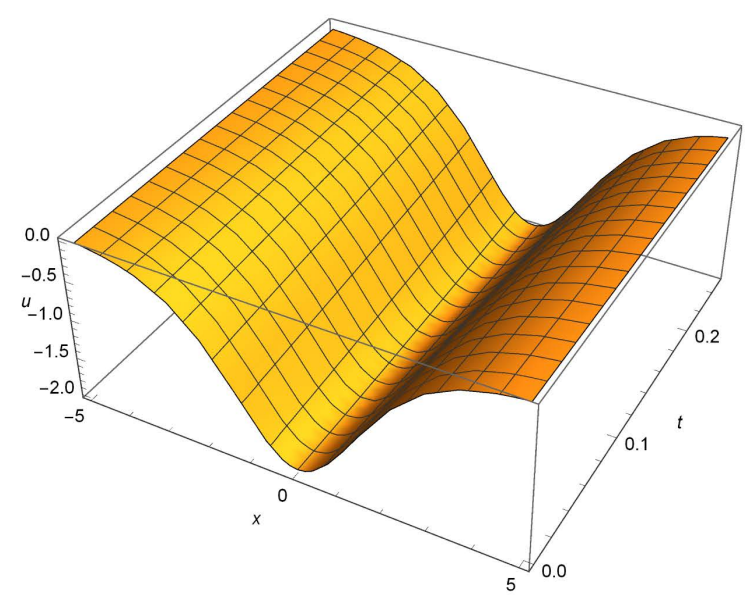

(a)

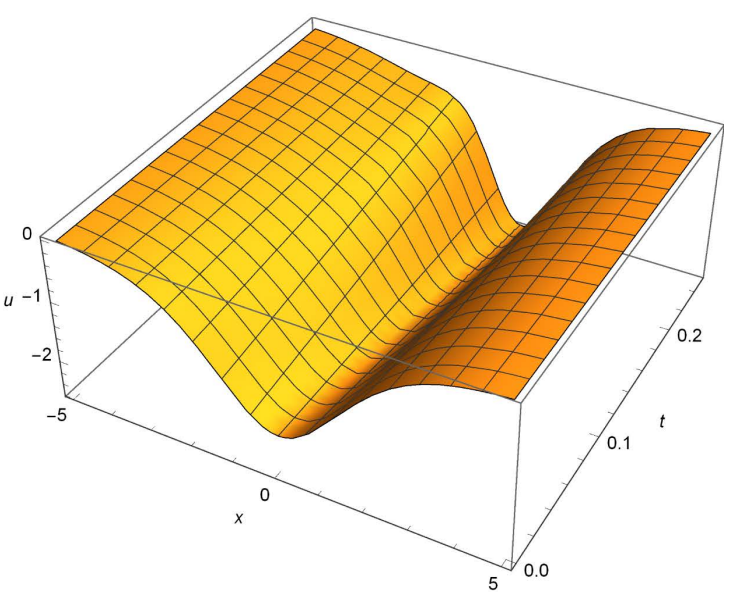

(b)

Figure 1. The surface $u(x, t)$ of mCH equation at different times. (a) Exact solution; (b) Approximate solution.

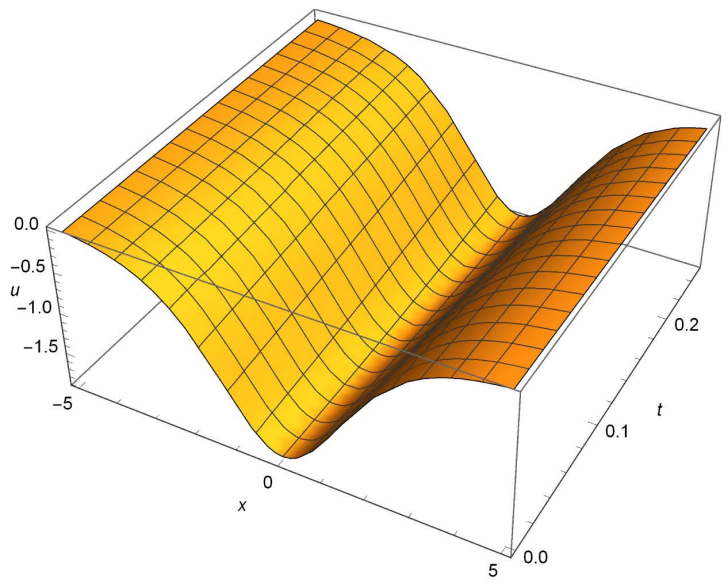

(a)

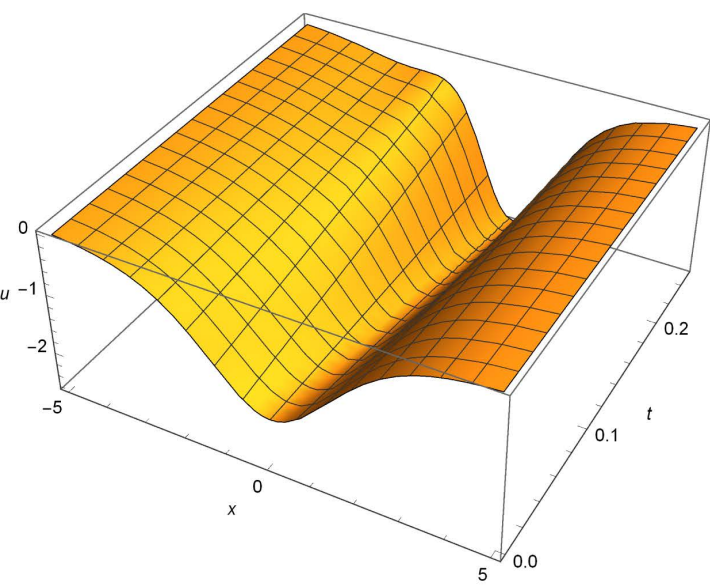

(b)

Figure 2. The surface $u(x, t)$ of mDP equation at different times. (a) Exact solution; (b) Approximate solution.

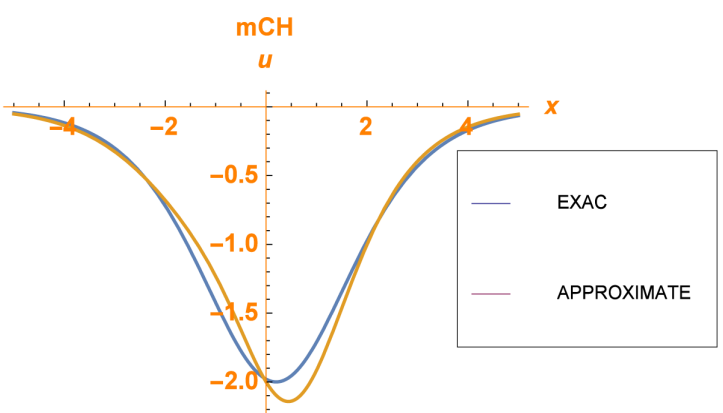

(a)

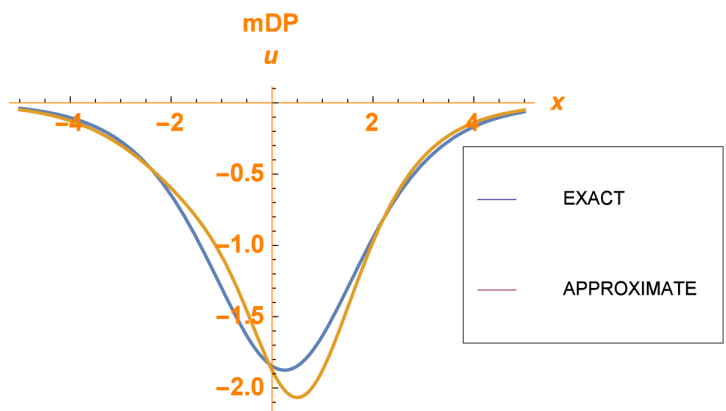

(b)

Figure 3. The curve of $u(x, t)$. (a) Exact and VHPM of mCH equation; (b) Exact and VHPM of mDP equation.

\section{Acknowledgements}

We thank the editor and the referee for their comments. Many thanks to University of Zakho for supporting this work. 


\section{References}

[1] Adomian, G. (1976) Nonlinear Stochastic Differential Equations. Journal of Mathematical Analysis and Applications, 55, 441-452. http://dx.doi.org/10.1016/0022-247X(76)90174-8

[2] Adomian, G. (1991) A Review of the Decomposition Method and Some Recent Results for Nonlinear Equations. Computers \& Mathematics with Applications, 21, 101-127. http://dx.doi.org/10.1016/0898-1221(91)90220-X

[3] Liao, S.J. (2004) On the Homotopy Analysis Method for Nonlinear Problems. Applied Mathematics and Computation, 147, 499-513. http://dx.doi.org/10.1016/S0096-3003(02)00790-7

[4] Abbasbandy, S. (2009) Solitary Wave Solutions to the Modified Form of Camassa Holm Equation by Means of the Homotopy Analysis Method. Chaos, Solitons and Fractals, 39, 428-435. http://dx.doi.org/10.1016/j.chaos.2007.04.007

[5] He, J.H. (1998) Approximate Solution of Nonlinear Differential Equations with Convolution Product Nonlinearities. Computer Methods in Applied Mechanics and Engineering, 167, 69-73. http://dx.doi.org/10.1016/S0045-7825(98)00109-1

[6] He, J.H. (1999) Variational Iteration Method a Kind of Non-Linear Analytical Technique: Some Examples. International Journal of Non-Linear Mechanics, 34, 699-708. http://dx.doi.org/10.1016/S0020-7462(98)00048-1

[7] He, J.H. (1999) Homotopy Perturbation Technique. Computer Methods in Applied Mechanics and Engineering, 178, 257-262. http://dx.doi.org/10.1016/S0045-7825(99)00018-3

[8] He, J.H. (2005) Application of Homotopy Perturbation Method to Nonlinear Wave Equations. Chaos, Solitons Fractals, 26, 695-700. http://dx.doi.org/10.1016/j.chaos.2005.03.006

[9] Rashidi, M.M., Ganji, D.D. and Dinarvand, S. (2009) Explicit Analytical Solutions of the Generalized Burger and Burger Fisher Equations by Homotopy Perturbation Method. Numerical Methods for Partial Differential Equations, 25, 409-417. http://dx.doi.org/10.1002/num.20350

[10] Rashidi, M.M., Freidoonimehr, N., Hosseini, A., Anwar Bg, O. and Hung, T.K. (2014) Homotopy Simulation of Nanofluid Dynamics from a Non-Linearly Stretching Isothermal Permeable Sheet with Transpiration. Meccanica, 49, 469-482. http://dx.doi.org/10.1007/s11012-013-9805-9

[11] Fadhil, H.E., Manaa, S.A., Bewar, A.M. and Majeed, A.Y. (2015) Variational Homotopy Perturbation Method for Solving Benjamin-Bona-Mahony Equation. Applied Mathematics, 6, 675-683. http://dx.doi.org/10.4236/am.2015.64062

[12] Olusola, E. (2013) New Improved Variational Homotopy Perturbation Method for Bratu-Type Problems. American Journal of Computational Mathematics, 3, 110-113. http://dx.doi.org/10.4236/ajcm.2013.32018

[13] Wazwaz, A.M. (2006) Solitary Wave Solutions for Modified Forms of Degasperis-Procesi and Camassa-Holm Equations. Physics Letter A, 352, 500-504.

[14] Wu, G.C. (2013) Challenge in the Variational Iteration Method-A New Approach to Identification of the Lagrange Multi-Pliers. Journal of King Saud University—Science, 25, 175-178. http://dx.doi.org/10.1016/j.jksus.2012.12.002

[15] He, J.H. (1997) A New Approach to Nonlinear Partial Differential Equations. Communications in Nonlinear Science and Numerical Simulation, 2, 230-235. http://dx.doi.org/10.1016/S1007-5704(97)90007-1

[16] Zhang, B.-G. and Li, S.-Y. (2008) Homotopy Perturbation Method for Modified Camassa-Holm and Degasperis-Procesi Equation. Physics Letter A, 372, 1867-1872.

[17] Yildirim, A. (2010) Variational Iteration Method for Modified Camassa-Holm and Degasperis-Procesi Equations. International Journal for Biomedical Engineering, 26, 266-272. 